\title{
Evaluation of parasitological and molecular techniques for the diagnosis and assessment of cure of schistosomiasis mansoni in a low transmission area
}

\author{
Liliane Maria Vidal Siqueira ${ }^{1 /+}$, Luciana Inácia Gomes², Edward Oliveira ${ }^{2}$, \\ Eduardo Ribeiro de Oliveira ${ }^{2}$, Áureo Almeida de Oliveira', Martin Johannes Enk ${ }^{3}$, \\ Nídia Figueiredo Carneiro", Ana Rabello², Paulo Marcos Zech Coelho'
}

'Laboratório de Esquistossomose 2Laboratório de Pesquisas Clínicas, Centro de Pesquisas René Rachou-Fiocruz, Belo Horizonte, MG, Brasil ${ }^{3}$ Instituto Evandro Chagas, Ananindeua, PA, Brasil ${ }^{4}$ Centro de Controle de Zoonoses, Montes Claros, MG, Brasil

This study evaluated parasitological and molecular techniques for the diagnosis and assessment of cure of schistosomiasis mansoni. A population-based study was performed in 201 inhabitants from a low transmission locality named Pedra Preta, municipality of Montes Claros, state of Minas Gerais, Brazil. Four stool samples were analysed using two techniques, the Kato-Katz ${ }^{\mathbb{}}$ (KK) technique (18 slides) and the TF-Test ${ }^{\mathbb{B}}$, to establish the infection rate. The positivity rate of $18 \mathrm{KK}$ slides of four stool samples was 28.9\% (58/201) and the combined parasitological techniques (KK+TFTest ${ }^{\mathbb{R}}$ ) produced a $35.8 \%$ positivity rate (72/201). Furthermore, a polymerase chain reaction (PCR)-ELISA assay produced a positivity rate of $23.4 \%$ (47/201) using the first sample. All 72 patients with positive parasitological exams were treated with a single dose of Praziquantel ${ }^{\circledR}$ and these patients were followed-up 30, 90 and 180 days after treatment to establish the cure rate. Cure rates obtained by the analysis of $12 \mathrm{KK}$ slides were $100 \%, 100 \%$ and $98.4 \%$ at 30,90 and 180 days after treatment, respectively. PCR-ELISA revealed cure rates of $98.5 \%, 95.5 \%$ and $96.5 \%$, respectively. The diagnostic and assessment of cure for schistosomiasis may require an increased number of KK slides or a test with higher sensitivity, such as PCR-ELISA, in situations of very low parasite load, such as after therapeutic interventions.

Key words: Schistosoma mansoni - schistosomiasis - diagnosis - control - low transmission area - Brazil

Approximately 200-300 million people worldwide are infected by one of the five main species of Schistosoma. Of the infected, 120 million people are symptomatic and 20 million are afflicted with a severe form of the disease. Approximately 779 million people are at risk of contracting the disease (Steinmann et al. 2006) and 280,000 deaths per year are attributed to this disease and its complications (Van der Werf et al. 2003).

Schistosomiasis is an important public health problem and the development of simple, low cost and efficient diagnostic methods that may be used on a mass scale, especially in field work, is of fundamental interest. However, decisions about individual treatment, the study of morbidity due to schistosomiasis, the rapid identification of communities at risk of infection, evaluations of cure or reinfection after chemotherapy and the monitoring of control programs are actions that depend primarily on the results of more accurate and effective diagnostic tests (Utzinger 1999).

The conventional diagnosis of $S$. mansoni infection is performed using the microscopic detection of parasite eggs in faeces. The Kato-Katz (KK) technique is rec-

doi: 10.1590/0074-02760140375

Financial support: FAPEMIG, CAPES, CNPq, FIOCRUZ

+Corresponding author: 1iliane_siqueira@cpqrr.fiocruz.br

Received 10 October 2014

Accepted 26 January 2015 ommended because it is quantitative, low cost and easy to perform (WHO 1993). However, difficulties arise in individuals with low worm burden because of the small number of eggs shed in the faeces, daily variations of oviposition by female worms and only a small amount of sample is examined in a single slide, approximately 41.7 $\mathrm{mg}$ of faeces (Kongs et al. 2001). Many schistosomiasis control programs adopted this procedure, which results in an underestimation of true disease prevalence, particularly in low transmission areas and individuals with low worm burdens (Enk et al. 2008). It is scientifically well demonstrated that the presence of $S$. mansoni eggs in a faeces sample varies more on different days than between various slides, which indicates that the results of a larger number of faecal examinations would be ideal to more accurately evaluate infection prevalence (Utzinger et al. 2001). Therefore, the possibility of false negative results cannot be excluded by negative stool examinations performed using only a few samples and slides.

Advances in disease diagnosis are not satisfactory despite increased awareness of the disease. This decrement is reflected in the effectiveness of control programs, which has become a major public health challenge that requires innovative and more effective strategies, such as the use of alternative diagnostic methodologies that would enable estimates of prevalence rates that are closer to reality (Enk et al. 2008).

Assays for the detection of antibodies and antigens are promising strategies that are complementary to the traditional parasitological examination. These methods are auxiliary assays that can determine therapeutic re- 
sponses and the persistence of infection, but they are not used in population surveys, because sophisticated laboratory infrastructure is required and the majority of tests are in the validation process. Moreover, diagnostic strategies must be combined when these assays are used in low endemic areas to obtain a better estimate of the infection rate of Schistosoma in populations that are at risk of the disease and evaluate the effectiveness of interventions, such as chemotherapy (Cavalcanti et al. 2013).

Commercially available immunological tests include ELISA kits and indirect haemagglutination for the detection of antibodies. Recently, the Point-of-Care (POC$\left.\mathrm{CCA}^{\circledR}\right)$ test was evaluated to detect circulating cathodic antigens in urine samples (Rapid Medical Diagnostics, South Africa) and this test showed better sensitivity than the KK technique (Colley et al. 2013).

Molecular methods are useful for the detection of low parasitic loads and these tests are highly sensitive for the laboratory diagnosis of infectious diseases. Recent DNA detection assays are drawing attention because their robustness, which makes these assays applicable for community and individual diagnoses of schistosomiasis mansoni. The polymerase chain reaction (PCR) technique is based on the amplification of a highly repetitive DNA sequence for the diagnosis of Schistosoma mansoni infection in snails (Hamburger et al. 1991) and it was adapted for the diagnosis of schistosomiasis mansoni in human faeces by Pontes et al. (2002). This work standardised and evaluated the technique in endemic areas and it detected 1 fg of $S$. mansoni DNA. The specificity of this method was demonstrated by the absence of the DNA of other helminths (Ascaris lumbricoides, Ancylostoma duodenale, Necator americanus, Taenia solium and Trichuris trichiura), which commonly infect people in endemic areas. However, this technique requires several steps after DNA amplification, including gel electrophoresis, which limits the number of samples that can be conveniently analysed.

The same group of researchers developed a PCR-ELISA system for the detection of Schistosoma DNA in human faeces as an alternative approach to the diagnosis of light infections (Gomes et al. 2010). This system permits the detection of DNA amplified by PCR using an ELISA platform, which measures colorimetric intensity in a microplate reader. The proposed PCR-ELISA approach was used for the diagnosis of a population in an endemic area in Brazil and 30\% were found to be infected compared with the $18 \%$ found using microscopic faecal examination. The genus specificity of PCR-ELISA was assessed using purified DNA from S. mansoni, Schistosoma haematobium, Schistosoma bovis, Schistosoma intercalatum, Schistosoma japonicum, Schistosoma magrebowiei and Schistosoma rhodaini adult worms. The advantage of PCR-ELISA compared with conventional PCR is that the combined technique uses standard equipment that is widely used for the processing of ELISAs and the reagents are easy to obtain commercially.

Therefore, this study evaluated the diagnostic performance of the KK technique plus the TF-Test ${ }^{\mathbb{B}}$ and the molecular assay PCR-ELISA for the laboratory diagnosis of schistosomiasis mansoni and assessment of cure in a low transmission area.

\section{SUBJECTS, MATERIALS AND METHODS}

Study area and population - This study was performed in 2009 in the communities of Buriti Seco and Morro Grande, which pertain to Pedra Preta, a little village in a schistosomiasis endemic area of the rural region of Montes Claros, state of Minas Gerais, Brazil. This area was chosen because the population shows a low migration index (fixed resident population) and the infection rate in 2005 when the last treatment occurred was $12 \%$ according to data provided by the Control Centre of Diseases of Montes Claros. The populations of the two communities were included in this study, which included 201 participants, 93 women and 108 men, aged between one-96 years.

Diagnostic techniques - An in depth analysis of stool samples, including a combination of parasitological examinations, was performed before treatment to establish the infection rate. This initial analysis was called the "Reference Test". Four stool samples from all participants were collected and analysed using the Kato-Katz ${ }^{\mathbb{B}}$ technique [Biomanguinhos-Oswaldo Cruz Foundation (Fiocruz), state of Rio de Janeiro, Brazil] and the commercial test TF-Test ${ }^{\mathbb{B}}$ (Immunoassay Com Ind Ltda, Brazil) as described by Siqueira et al. (2011). The PCR-ELISA assay was used on $500 \mathrm{mg}$ of the first stool sample of each participant for comparison with the parasitological tests and evaluation of diagnostic accuracy (Fig. 1).

Kato-Katz ${ }^{\circledR}$ - The KK test was applied using 18 slides that were prepared as follows: 12 slides of the first sample and two slides of the second, third and fourth samples were analysed for a total of $750 \mathrm{mg}$ of faeces for each participant (18 x $41.7 \mathrm{mg})$.

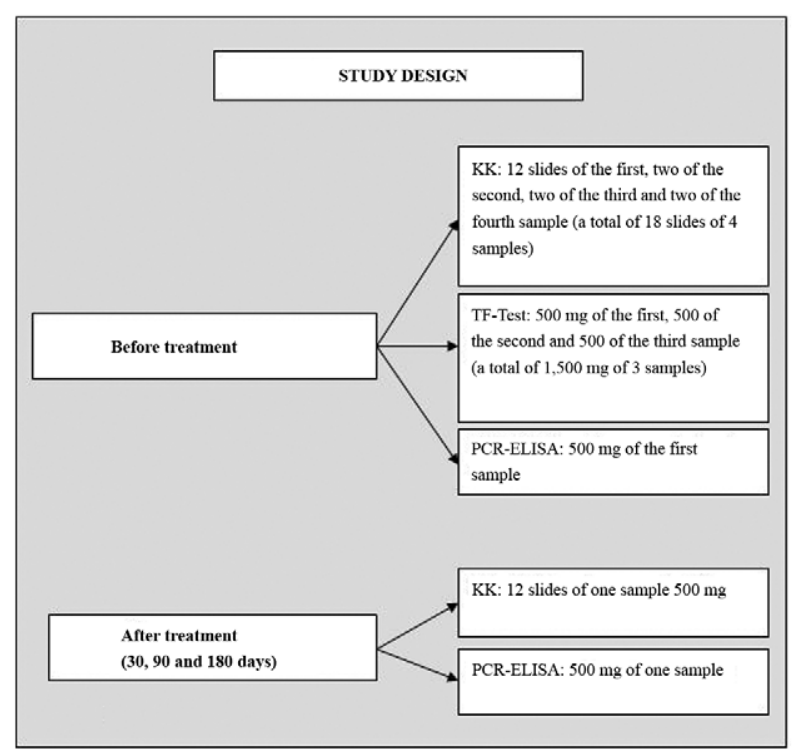

Fig. 1: flowchart of stool examinations according to different diagnostic techniques for establishing infection rate and the follow-up of positives after treatment. KK: Kato-Katz; PCR: polymerase chain reaction. 
TF-Test $t^{\circledR}$ - The TF-Test ${ }^{\circledR}$ was performed on the first three samples using portions of $500 \mathrm{mg}$, totalling 1,500 $\mathrm{mg}$ of faeces, which was processed with $3 \mathrm{~mL}$ of ethyl acetate and one drop of detergent and centrifuged for 2 $\mathrm{min}$ at $500 \mathrm{~g}$.

PCR-ELISA assay - DNA from 500-mg stool samples was isolated using the commercial kit QIAamp ${ }^{\circledR}$ DNA Stool Mini Kit, following the manufacturer's recommendations (Qiagen GMbH, Germany). A 121-bp repetitive sequence of $S$. mansoni (Hamburger et al. 1991) was amplified using a forward biotinylated primer (5'GATCTGAATCCGACCAACCG-3') and reverse primer (5'-ATATTAACGCCCACGCTCTC-3'), according to the protocols described by Gomes et al. (2010). The amplicons were detected in an ELISA plate sensitised with streptavidin using a fluorescein 5'-labeled probe (5'-TGGTTTCGGAGATACAACGA-3') with available reagents in the commercial kit PCR Plate Detection, according to the manufacturer's recommendations (Sigma, USA). All experiments were performed in duplicate and data represent the mean values. Positive controls extracted from $S$. mansoni eggs and negative controls (water and negative control of PCR) were added to each PCR-ELISA assay. The receiver operating characteristic curve (ROC) determined the cut-off value of 0.116 . Individuals with a mean absorbance above the cut-off were considered positive. The human beta-actin gene was amplified using the primers Acol (5'-ACCTCATGAAGATCCTCACC-3'), biotinylated in the 5' region and Aco2 (5'-CCATCTCTTGCTCGAAGTCC-3') as a control for variations in the efficiency of DNA extraction and PCR-amplification. Products were detected using a fluorescein 5 '-labeled probe (5'-TCTCCTTAATGCACGCACG- $\left.3^{\prime}\right)$ in the PCRELISA system as described by Gomes et al. (2010).

One stool sample of each treated participant was analysed after treatment to establish the cure rate using the KK technique examining 12 slides per sample and the PCR-ELISA assay, which used $500 \mathrm{mg}$ of the same stool sample.

Comparisons of different diagnostic approaches Diagnostic parameters were calculated using three different approaches for comparison; (i) two-slide KK examination, which is the current recommendation of the Brazilian Ministry of Health for the national schistosomiasis control program, (ii) 12-slide KK examinations to observe the diagnostic parameters using the same amount of faecal material and (iii) the Reference Test described above as an approximation of the real infection rate to evaluate test performance.

Statistical analysis - The software OpenEpi, v.3.03 (Dean et al. 2014) was used for statistical analyses. Co-positivity and co-negativity values were calculated with $95 \%$ confidence intervals (CI) for each of the three approaches. The level of diagnostic agreement between different diagnostic techniques was determined using the kappa coefficient. Landis and Koch (1977) demonstrated poor concordance when this coefficient was lower than 0.20 , weak concordance between 0.21-0.40, moderate concordance between 0.41-0.60, good concordance between 0.61- 0.80 and excellent concordance when higher than 0.81 .
Treatment of positives - All participants who were positive for schistosomiasis as detected using the parasitological techniques were treated with a single dose of $60 \mathrm{mg} / \mathrm{kg}$ praziquantel for children and $50 \mathrm{mg} / \mathrm{kg}$ for adults. Infections with other helminths were treated using a single dose of $400 \mathrm{mg}$ albendazole, as proscribed by the Brazilian Ministry of Health.

Cure assessment - Cure assessments were performed 30, 90 and 180 days post-treatment. Sixty-nine of the 72 positive patients were followed up 30 days after treatment, 67 patients were followed up after 90 days and 61 patients were followed up after 180 days. One stool sample from each participant was analysed using the KK technique (12 slides per sample) and the PCR-ELISA assay, which was applied to $500 \mathrm{mg}$ of the same stool sample.

Ethics - The Ethical Research Committee of the Research Centre René Rachou/Fiocruz (CEPSH/CPQRR 03/2008) and the National Brazilian Ethical Board (Scientific Opinion 784/2008, CONEP 14886) approved this study. The objectives of this study were presented and explained to all participants and written consent was obtained from each individual, who signed a form prior to enrolment in this study.

\section{RESULTS}

Seventy-two of the 201 stool samples examined were positive using the Reference Test. Two-slide KK examination of the same sample, which is the current recommendation of the Brazilian Ministry of Health for the national schistosomiasis control program, detected 16 positives ( $8 \%$ ), examination of $12 \mathrm{KK}$ slides detected 29 positives (14.4\%), $18 \mathrm{KK}$ slides detected 58 positives (28.9\%), 32 positives $(15.9 \%)$ were detected using the TFTest $^{\mathbb{R}}$ and 47 positives $(23.4 \%)$ were detected using PCRELISA (Fig. 2). The comparison of PCR-ELISA with two KK slides, which corresponded to $83.4 \mathrm{mg}$ of faeces, revealed that the co-positivity of PCR-ELISA was $93.75 \%$ (CI: 71.67-98.89\%) and the co-negativity was $82.7 \%$ (CI: 76.6-87.47). The comparison with $12 \mathrm{KK}$ slides of the same sample, which corresponded to $500 \mathrm{mg}$ of faeces (41.7 mg x $12=500 \mathrm{mg}$ ), demonstrated a co-positivity of PCR-ELISA of $89.66 \%$ (CI: 73.61-96.42\%) and a conegativity of $87.8 \%$ (CI: 82.06-91.87\%) (Table I).

Out of 47 positives detected by PCR-ELISA using $500 \mathrm{mg}$ of the first sample, 12 were not identified by the Reference Test. Thirty-seven positives only were detected by the Reference Test, however the first assay was achieved with a single sample and the parasitological combination with four samples.

The kappa index was calculated to evaluate agreement among the methods. Two KK slides showed moderate agreement with PCR-ELISA (kappa index of 0.405) and 12 slides showed a substantial agreement (kappa index of 0.616) (Table II). The PCR-ELISA assay showed moderate agreement with the Reference Test (kappa index of 0.426) (Table III).

The cure rate 30 days after treatment using the KK technique was $100 \%$ and one positive individual was detected using PCR-ELISA (cure rate of 98.5\%). Three positive individuals were detected using PCR-ELISA 90 days 


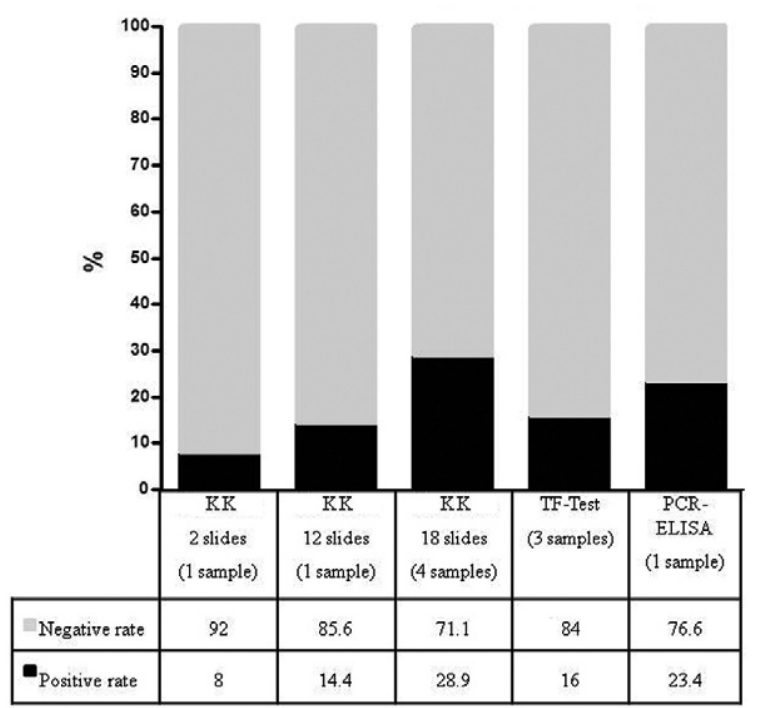

Fig. 2: diagnostic performance of parasitological and molecular techniques using stool samples, Pedra Preta, municipality of Montes Claros, state of Minas Gerais, Brazil, 2009. KK: Kato-Katz; PCR: polymerase chain reaction.

TABLE I

Results of co-positivity and co-negativity for polymerase chain reaction (PCR)-ELISA in relation to two Kato-Katz (KK) slides and in direct comparison with $12 \mathrm{KK}$ slides for the detection of infection with Schistosoma mansoni

\begin{tabular}{lcc}
\hline $\begin{array}{l}\text { KK } \\
\text { positivity rates } \\
\%(\mathrm{n} / \mathrm{n})\end{array}$ & \multicolumn{1}{c}{$\begin{array}{c}\text { PCR-ELISA } \\
\%(\mathrm{n} / \mathrm{n})\end{array}$} \\
\cline { 2 - 3 } $\begin{array}{l}8(16 / 201) \\
(2 \text { slides }=83.4 \mathrm{mg})\end{array}$ & $93.7(15 / 16)$ & $82.7(153 / 185)$ \\
$\begin{array}{l}\text { Co-positivity } \\
(12 \text { slides }=500 \mathrm{mg})\end{array}$ & $89.7(26 / 29)$ & $87.8(151 / 172)$ \\
\hline
\end{tabular}

after treatment (cure rate of 95.5\%), but these patients were not identified using analysis of $12 \mathrm{KK}$ slides. The cure rate 180 days after treatment was $98.4 \%$ using KK (1 out of 61 ) and $96.5 \%$ using PCR-ELISA (2 out of 61 ), with a final cure rate of $95.1 \%$ (3 out of 61) (Table IV).

\section{DISCUSSION}

Evaluations of PCR-ELISA assay showed promising results. This assay proved a valuable tool for the diagnosis of schistosomiasis despite its relatively high cost. PCR-ELISA can be applied to comparisons between diagnostic methods and it is very useful to confirm suspicious cases that were not detected using conventional parasitological methods and evaluate cure control after chemotherapeutic treatment.

The PCR technique is widely used for the diagnosis of several human diseases, but its application in neglected diseases, especially schistosomiasis, were explored only recently. Pontes et al. (2002) first described the use of PCR in faecal samples for the diagnosis of schistosomiasis. Some authors obtained high sensitivity and specificity (Pontes et al. 2003, Allam et al. 2009) compared to routine parasitological examinations, such as the KK technique (Katz et al. 1972).

The positivity rate detected using PCR-ELISA was $23.4 \%$ in the present study, which was higher than the $14.4 \%$ using KK technique (12 slides). However, three participants who exhibited shedding eggs in faeces (diagnosed using the KK technique) were not identified using PCR-ELISA, which may be explained by the absence of eggs in the samples examined by PCR assay, the presence of Taq DNA polymerase enzyme inhibitors or failure in extraction, although the positive internal control (human beta-actin gene) was detected in these samples. Further, the DNA from these negative PCR-ELISA samples were diluted 1:10, 1:20 and 1:40 and assayed again to exclude the possibility of inhibiting the reaction, but the results were identical to the initial assay.

PCR-ELISA detected 20 positive cases that were not identified using the KK technique (12 slides). Our data corroborate the findings of Allam et al. (2009) who

TABLE II

Comparison and agreement between results detected by the polymerase chain reaction (PCR)-ELISA in relation to Kato-Katz (KK) method using two and 12 slides of the same stool sample

\begin{tabular}{|c|c|c|c|c|c|c|c|c|}
\hline & \multicolumn{4}{|c|}{$\begin{array}{c}\text { KK } \\
(2 \text { slides }=83.4 \mathrm{mg})\end{array}$} & \multicolumn{4}{|c|}{$\begin{array}{c}\mathrm{KK} \\
(12 \text { slides }=500 \mathrm{mg})\end{array}$} \\
\hline & & Positive & Negative & Total & & Positive & Negative & Total \\
\hline \multirow{2}{*}{$\begin{array}{l}\text { PCR-ELISA } \\
(500 \mathrm{mg} \text { of } \\
\text { the } 1 \mathrm{st} \text { sample) }\end{array}$} & Positive & 15 & 32 & 47 & Positive & 26 & 21 & 47 \\
\hline & Negative & 1 & 153 & 154 & Negative & 3 & 151 & 154 \\
\hline \multirow[t]{2}{*}{ Total } & & 16 & 185 & 201 & & 29 & 172 & 201 \\
\hline & \multicolumn{4}{|c|}{ Kappa index: 0.405} & \multicolumn{4}{|c|}{ Kappa index: 0.616} \\
\hline
\end{tabular}


TABLE III

Comparison and agreement between results detected by the Reference Test ${ }^{a}$ and polymerase chain reaction (PCR)-ELISA ${ }^{b}$

\begin{tabular}{lcccc}
\hline & & \multicolumn{3}{c}{ Reference Test } \\
\cline { 2 - 4 } & & Positive & Negative & Total \\
\hline $\begin{array}{l}\text { PCR-ELISA } \\
\text { (500 mg of } \\
\text { the 1st sample })\end{array}$ & Positive & 35 & 12 & 47 \\
& Negative & 37 & 117 & 154 \\
\hline Total & & 72 & 129 & 201 \\
\cline { 3 - 4 } & & \multicolumn{4}{c}{ Kappa $=0.426$} \\
\hline
\end{tabular}

$a$ : four samples $=18$ slides of Kato-Katz + TF-Test ${ }^{\mathbb{}} ; b$ : single sample.

showed that PCR detected 23\% positive cases amongst negative samples for $S$. mansoni using the KK technique from individuals living in a hypoendemic area. Similar results were found by Rabello et al. (2002) and by Pontes et al. (2003) using conventional PCR and visualisation on gel electrophoresis. These authors reported that 16 out of 194 participants exhibited positive PCR, but did not present eggs in faeces, whereas two positive participants using the KK method were negative using PCR. Pontes et al. (2003) explained that the positive cases that were not detected using PCR could occur during the DNA amplification reaction as a result of various factors, such as amplification inhibition by faecal compounds, DNA degradation during transportation from the field to the laboratory or the absence of eggs in the sample examined.

A study by Gomes et al. (2010) demonstrated that PCR-ELISA assay analysis of stool samples from 206 individuals from an endemic area compared with 12 KK slides from a single sample revealed a sensitivity of $97.4 \%$ and specificity of $85.1 \%$, which are higher than the values in our study. This difference may be explained by factors that are inherent in the experimental procedures, which may generate errors related to the detection of parasite DNA, its degradation during biological sample collection or absence in the sample analysed. The need for well-trained technicians who rigorously apply quality control measures at all stages of the reaction should be stressed to avoid false-negative or false-positive results from contamination.

Molecular methods may suffer a loss of standardisation (reproducibility) and strength when "in house" techniques are used. These criteria are required for the possible application of these methods in control programs (Gomes et al. 2009). Faecal samples are difficult to use for molecular analyses. The commercial kit QIAamp ${ }^{\circledR}$ DNA Stool Mini Kit was used in this study for DNA extraction from stool to avoid methodological interferences in the procedure. Physical methods (e.g., separation of rooms and materials and the use of laminar flow hood with ultraviolet light) were utilised throughout the experiment to minimise the chances of sample contamination. This technique requires laboratory infrastructure and a more complex operating engagement. Therefore, it is indicated for diagnosis of Schistosoma spp in special situations, when high sensitivity and specificity are required and infrastructure is available (Rabello et al. 2002).

A greater number of parasitic infections with low viral load are detected using diagnostic methods with increased sensitivity. Seventy of the 72 (97.2\%) individuals infected by $S$. mansoni presented low worm burden [1-100 eggs per gram (epg)], one individual showed medium parasite burden (156 epg) and another patient showed a high intensity of infection (555 epg) as evaluated using the Reference Test.

The assessment of cure after treatment using the KK technique and PCR-ELISA assay showed a decrease of infected individuals. Different reasons may explain the differences in cure rates between these techniques. For example, DNA from dead worms or eggs remaining in tissues can be excreted in faeces for a period of time after treatment. Another reason is that the sensitivity of the PCR-ELISA for the detection of low parasite burdens is greater than the KK technique (Wichmann et al. 2013). The lower cure rate six months after treatment may be explained by the temporary interruption of the oviposi-

TABLE IV

Assessment of cure 30, 90 and 180 days after treatment by the Kato-Katz (KK) and polymerase chain reaction (PCR)-ELISA techniques

\begin{tabular}{lccc}
\hline & \multicolumn{3}{c}{$\begin{array}{c}\text { Cure rate } \\
\%(\mathrm{n} / \mathrm{n})\end{array}$} \\
\cline { 2 - 4 } Techniques & 30 days & 90 days & 180 days \\
\hline $\begin{array}{l}\text { KK } \\
(12 \text { slides }=500 \mathrm{mg})\end{array}$ & $100(69 / 69)$ & $100(67 / 67)$ & $98.4(60 / 61)$ \\
$\begin{array}{l}\text { PCR-ELISA } \\
(500 \mathrm{mg})\end{array}$ & $98.5(68 / 69)$ & $95.5(64 / 67)$ & $96.5(59 / 61)$ \\
\hline
\end{tabular}


tion by female worms as a result of treatment and a resumption of oviposition at a later time. These individuals also may have been re-infected because of the constant exposure they may suffer in the endemic areas.

Managers of control programs in areas of low transmission must choose between the KK technique with various samples or PCR-ELISA assay to detect the largest number of infected individuals using a single sample. Notably, the first technique is less expensive, but the latter technique is more accurate. The cost of PCR reagents is US \$10 per faecal sample (Gomes et al. 2010), but these costs are greatly reduced when the technique is utilised on a large scale. PCR also provides good precision (reproducibility and repeatability), is genus-specific and may estimate worm burden, which correlates with the values of epg of faeces determined using the KK technique.

The results obtained using PCR-ELISA support the development of a promising diagnostic method that only uses a faecal sample. This technique represents an important step forward to support studies on epidemiology and disease control, especially when the cost would be reduced by the use of industrial scale production. The assessment of cure in situations of very low parasite load, such as after therapeutic interventions, may require increased numbers of KK slides or a test with higher sensitivity than PCRELISA. This study also demonstrated a low positivity rate during follow-up after treatment, which indicates that an in-depth diagnostic survey is more effective and it may likely prevent successive treatments.

\section{ACKNOWLEDGEMENTS}

To Vera de Paula Ribeiro, from the Laboratory of Schistosomiasis, CPqRR, to referees review of the English, for the cooperation of the technical team of the Control Centre of Diseases of Montes Claros, and to the population of Pedra Preta, for their collaboration during the field work.

\section{REFERENCES}

Allam AF, Kader O, Zaki A, Shehab AY, Farag HF 2009. Assessing the marginal error in diagnosis and cure of Schistosoma mansoni in areas of low endemicity using Percoll and PCR techniques. Trop Med Int Health 14: 316-321.

Cavalcanti MG, Silva LF, Peralta RHS, Barreto MGM, Peralta JM 2013. Schistosomiasis in areas of low endemicity: a new era in diagnosis. Trends Parasitol 29: 75-82.

Colley DG, Binder S, Campbell C, King CH, Tchuem Tchuentė LA, N'Goran EK, Erko B, Karanja DM, Kabatereine NB, van Lieshout L, Rathbun S 2013. A five-country evaluation of a Point-ofCare circulating cathodic antigen urine assay for the prevalence of Schistosoma mansoni. Am J Trop Med Hyg 88: 426-432.

Dean AG, Sullivan KM, Soe MM 2014. OpenEpi: Open Source Epidemiologic Statistics for Public Health. Available from: OpenEpi.com.

Enk MJ, Lustosa Lima AC, Drummond SC, Schall VT, Coelho PMZ 2008. The impact of the number of stool samples on the prevalence, the infection intensity and the distribution of the infection with Schistosoma mansoni among a population in an area of low transmission. Acta Trop 108: 222-228.
Gomes LI, Marques LHS, Enk MJ, Coelho PMZ, Rabello A 2009. Further evaluation of an updated PCR assay for the detection of Schistosoma mansoni DNA in human stool samples. Mem Inst Oswaldo Cruz 104: 1194-1196.

Gomes LI, Marques LHS, Enk MJ, Oliveira MC, Coelho PMZ, Rabello A 2010. Developmente and evaluation of a sensitive PCRELISA system for detection of Schistosoma infection in feces. PLoS Negl Trop Dis 4: e664.

Hamburger J, Turetski T, Kapeller I, Deresiewicz R 1991. Highly repeated short DNA sequences in the genome of Schistosoma mansoni recognized by a species specific probe. Mol Biochem Parasitol 44: 73-80.

Katz N, Chaves A, Pellegrino J 1972. A simple device for quantitative stool thick-smear technique in schistosomiasis mansoni. Rev Inst Med Trop Sao Paulo 14: 397-400.

Kongs A, Marks G, Verlé P, Van der Stuyft P 2001. Limitations of Kato-Katz technique for evaluating S. mansoni infections. Trop Med Int Health 6: 163-169.

Landis JR, Koch GG 1977. The measurement of observer agreement for categorical data. Biometrics 33: 159-174.

Pontes LA, Dias-Neto E, Rabello A 2002. Detection by polymerase chain reaction of Schistosoma mansoni DNA in human serum and faeces. Am J Trop Med Hyg 66: 157-162.

Pontes LA, Oliveira MC, Katz N, Dias-Neto E, Rabello A 2003. Comparison of a polymerase chain reaction and the Kato-Katz technique for diagnosing infection with Schistosoma mansoni. Am J Trop Med Hyg 68: 652-656.

Rabello A, Pontes LA, Dias-Neto E 2002. Recent advances in the diagnosis of Schistosoma infection: the detection of parasite DNA. Mem Inst Oswaldo Cruz 97 (Suppl. I): 171-172.

Siqueira LMV, Coelho PMZ, de Oliveira AA, Massara CL, Carneiro NFF, Lima ACL, Enk MJ 2011. Evaluation of two coproscopic techniques for the diagnosis of schistosomiasis in a low-transmission area in the state of Minas Gerais, Brazil. Mem Inst Oswaldo Cruz 106: 844-850.

Steinmann P, Keiser J, Bos R, Tanner M, Utzinger J 2006. Schistosomiasis and water resources development: systematic review, meta-analysis and estimates of people at risk. Lancet Infect Dis 6: 411-425.

Utzinger J 1999. Novel approaches in the control of schistosomiasis: from rapid identification to chemoprophylaxis, $\mathrm{PhD}$ Thesis, University of Basel, Basel, $202 \mathrm{pp}$.

Utzinger J, Booth M, N'Goran EK, Muller I, Tanner M, Lengeler C 2001. Relative contribution of day-to-day and intra-specimen variation in faecal egg counts of Schistosoma mansoni before and after treatment with praziquantel. Parasitol 122: 537-544.

Van der Werf MJ, de Vlas SJ, Brooker S, Looman CW, Nagelkerke NJ, Habbema JD, Engels D 2003. Quantification of clinical morbidity associated with schistosome infection in sub-Saharan Africa. Acta Trop 89: 125-139.

Wichmann D, Poppert S, Von Thien H, Clerinx J, Dieckmann S, Jensenius M, Parola P, Richter J, Schunk M, Stich A, Zanger P, Burchard GD, Tannich E 2013. Prospective European-wide multicentre study on a blood based real-time PCR for the diagnosis of acute schistosomiasis. BMC Infect Dis 13: 55.

WHO - World Health Organization 1993. The control of schistosomiasis. Tech Rep Ser 830: 1-85. 